\title{
Noninvasive and invasive evaluation of pulmonary arterial pressure in highlanders
}

\author{
B.K. Kojonazarov*, B.Z. Imanov", T.A. Amatov", M.M. Mirrakhimov", R. Naeije ${ }^{\star}$, \\ M.R. Wilkins ${ }^{+}$and A.A. Aldashev*
}

ABSTRACT: The purpose of the present study was to evaluate Doppler echocardiography for the detection of pulmonary hypertension in high-altitude inhabitants.

In total, 60 (55 male) patients aged 18-71 yrs were recruited from an ECG screening programme applied to 1,430 inhabitants living at an altitude of 2,500-3,600 m in Kyrgyzstan. Of these, 44 met ECG criteria for right ventricular hypertrophy. All underwent Doppler echocardiography followed by a cardiac catheterisation within 7 days of arrival in Bishkek (Kyrgyzstan; altitude $760 \mathrm{~m}$ ). Pulmonary flow acceleration time and the maximum velocity of tricuspid regurgitation were measured.

Sufficient quality tricuspid regurgitant jets were recovered in only $28 \%$ of the patients. Therefore, pulmonary artery pressure was estimated from the pulmonary flow acceleration time, which was recovered in $100 \%$ of the patients. It was found that $37(62 \%)$ of the patients had pulmonary hypertension on echocardiography. Pulmonary hypertension was confirmed in 29 patients on catheterisation. Pulmonary hypertension was detected with $\mathbf{7 0} \%$ sensitivity and $88 \%$ specificity by echocardiography, as compared to $59 \%$ sensitivity and $81 \%$ specificity by ECG. The correlation coefficient between echocardiography and catheterisation studies was $r^{2}=0.78$.

It is concluded that a combination of ECG and echocardiography may be useful for screening high-altitude pulmonary hypertension.

KEYWORDS: ECG, echocardiography, high-altitude pulmonary hypertension, pulmonary circulation, right heart catheterisation

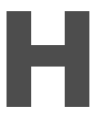

igh-altitude pulmonary hypertension (HAPH) is a public health problem in mountainous areas of the world. In Kyrgyzstan, the Tien-Shan and Pamir mountains occupy $\sim 90 \%$ of the territory and more than 200,000 people live $>2500 \mathrm{~m}$ above sea level. Previous studies have suggested that $4-6 \%$ of Kyrgyz highlanders with pulmonary hypertension develop right ventricular failure [1].

$\mathrm{HAPH}$ is characterised by increased pulmonary arterial pressure $(P \mathrm{pa})$ and pulmonary vascular remodelling involving all elements of the vessel wall, including endothelial and smooth muscle cells and fibroblasts [2-4]. Diagnosing the condition is dependent on accurate measurement of Ppa. Direct measurement by cardiac catheter remains the gold standard but there are practical problems with using this technique at altitude and it is unacceptable for routine screening. ECG and Doppler echocardiography assessments are alternative approaches but their sensitivity and specificity remain uncertain.
The purpose of the current study was to determine the value of Doppler echocardiography in screening for HAPH.

\section{METHODS AND MATERIALS Patients}

The study population was recruited from 1,430 ethnic Kyrgyz residents (aged 18-86 yrs) of four villages in the Naryn region (altitudes 2,500 3,000 m) and Ak-Say plateau (altitude 3,600 m) of Kyrgyzstan who underwent health screening by history, physical examination, blood pressure measurement, spirometry and ECG (fig. 1). Subjects were invited for further investigation by echocardiography and cardiac catheterisation in Bishkek (altitude $760 \mathrm{~m}$ ) if they met the following criteria. 1) ECG signs of right ventricular hypertrophy (ECG-RVH), defined later, without obvious cause and an otherwise normal medical examination. Alternatively, normal ECG and medical examination and in the same age group as subjects with ECG-RVH. 2) Willing to travel to Bishkek. In total, 63 subjects volunteered
AFFILIATIONS

*Institute of Molecular Biology and Medicine, and

${ }^{\#}$ National Center of Cardiology and Internal Medicine

Bishkek, Kyrgyzstan,

'Dept of Pathophysiology, Free

University of Brussels

Brussels, Belgium, and

${ }^{+}$Section of Experimental Medicine

and Toxicology, Imperial College

London, Hammersmith Hospital

London, United Kingdom.

CORRESPONDENCE

M.R. Wilkins

Section of Experimental Medicine

and Toxicology

Imperial College London

Hammersmith Hospital

Du Cane Road

London W12 ONN

UK

Fax: 442083832066

E-mail:m.wilkins@imperial.ac.uk

Received:

November 222005

Accepted after revision:

October 122006

STATEMENT OF INTEREST

None declared.

European Respiratory Journal

Print ISSN 0903-1936

Online ISSN 1399-3003 
but three were subsequently excluded on rescreening in Bishkek because of an atrial septal defect (two subjects) or coronary vascular disease (one subject). Of the remaining 60, none had evidence of coexisting disease (table 1). Forty-four (mean \pm SD aged $51 \pm 16 \mathrm{yrs}$ ) had ECG-RVH and 16 (aged $47 \pm 15 \mathrm{yrs}$ ) had a normal ECG. The ethics committee of the National Center of Cardiology and Internal Medicine (Bishkek, Kyrgyzstan) approved the study and all subjects gave informed consent.

\section{ECG}

Standard 12-lead ECG was performed at high altitude and repeated in Bishkek in the 60 highlanders volunteering for further investigation. RVH was diagnosed using one of the following patterns [5]: right axis deviation, defined as a frontal plane QRS axis of $\geqslant 90$ (pattern A); $\mathrm{R}$ wave in lead $\mathrm{V}_{1}$ of $\geqslant 5 \mathrm{~mm}$, an $\mathrm{R} / \mathrm{S}$ ratio of $>1$, and $\mathrm{S}>\mathrm{R}$ in $\mathrm{V}_{5}$ or $\mathrm{V}_{6}$ (pattern $\mathrm{B}$ ); a leftward shift in the transition zone (pattern $\mathrm{C}$ ).

\section{Doppler echocardiography}

Doppler echocardiography was performed using a commercially available portable Doppler echocardiograph (Sequoia 256; Acuson Corporation, Mountain View, CA, USA) and a transducer array of $2.5 \mathrm{MHz}$. Images were obtained from standard parasternal views (long axis and short axis) and an apical four-chamber view. The subjects were placed in the left

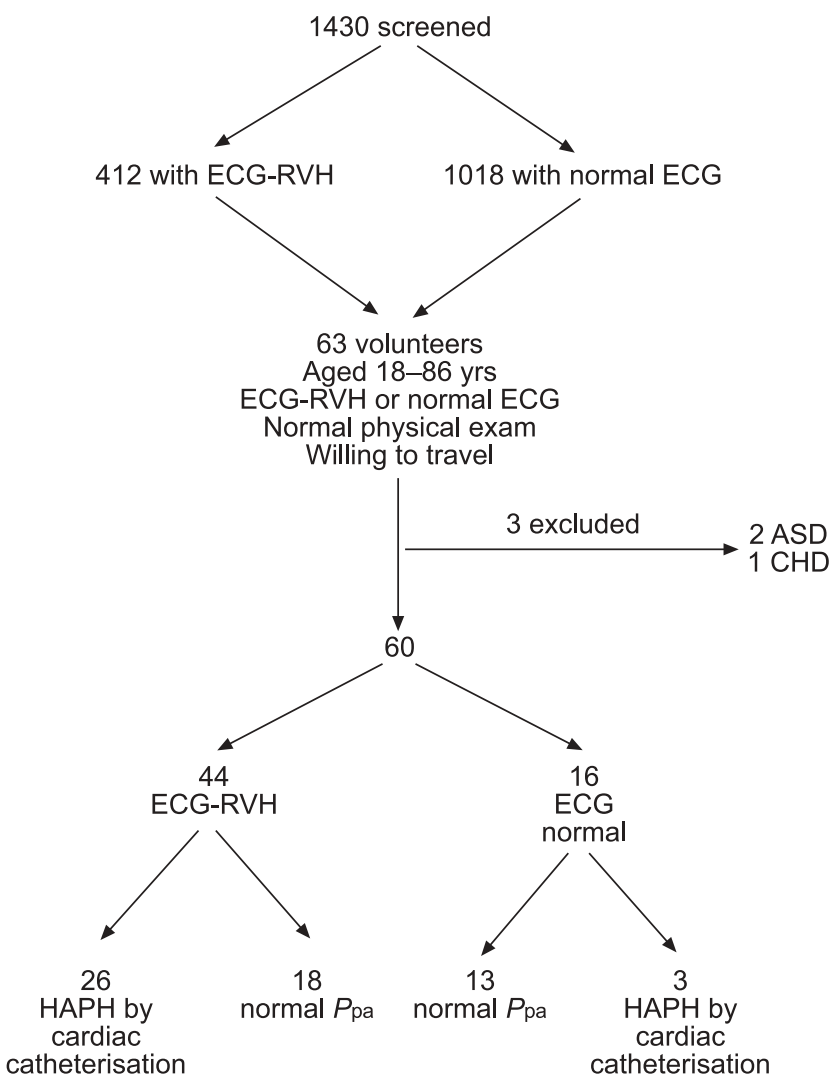

FIGURE 1. Flow of study subjects from screening to diagnosis of high-altitude pulmonary hypertension (HAPH) by cardiac catheterisation. ECG-RVH: right ventricular hypertrophy on ECG; Ppa: pulmonary artery pressure; ASD: atrial septal defect; CHD: coronary heart disease. lateral decubitus position for the parasternal views and in a supine position for the apical four-chamber view. Patients were investigated in the morning after $30 \mathrm{~min}$ of rest. Recordings were made on S-VHS videotape with simultaneous tracing of the ECG. The recordings were analysed by two independent echocardiographers, who were unaware of the clinical histories or cardiac catheter results. Right ventricle end-systolic and end-diastolic diameters were measured by Mmode echocardiography from the left parasternal short- and long-axis views. Continuous Doppler tricuspid regurgitant jets were recorded and analysed for the estimation of systolic $P$ pa [6]. A pulsed Doppler pulmonary blood flow velocity signal was sampled in the right ventricular outflow tract for the estimation of mean Ppa [7]. For this purpose, an acceleration time was defined as the time interval from the onset of forward flow in the pulmonary artery to the peak velocity of this flow [7]. HAPH was diagnosed if resting mean $P$ pa was $\geqslant 25 \mathrm{mmHg}$ [4]. A right ventricular anterior wall thickness $>0.5 \mathrm{~cm}$ was taken as evidence of RVH [8].

\section{Right heart catheterisation}

Right heart catheterisation was performed in Bishkek within $24 \mathrm{~h}$ of the echocardiography with a Swan-Ganz thermodilution catheter (Baxter Healthcare, Compton, UK) introduced via an internal jugular vein. The investigator was unaware of the echocardiography data. Patients were investigated in the supine position, in the morning after $30 \mathrm{~min}$ of rest. Baseline measurements consisted of systolic, diastolic and mean $P$ pa and pulmonary artery wedge pressure. Cardiac output was measured by continuous thermodilution with a Vigilance Monitor (Baxter Healthcare). Arterial oxygen saturation, ECG and blood pressure were monitored throughout. HAPH was diagnosed if resting mean $P$ pa was $\geqslant 25 \mathrm{mmHg}$ [4].

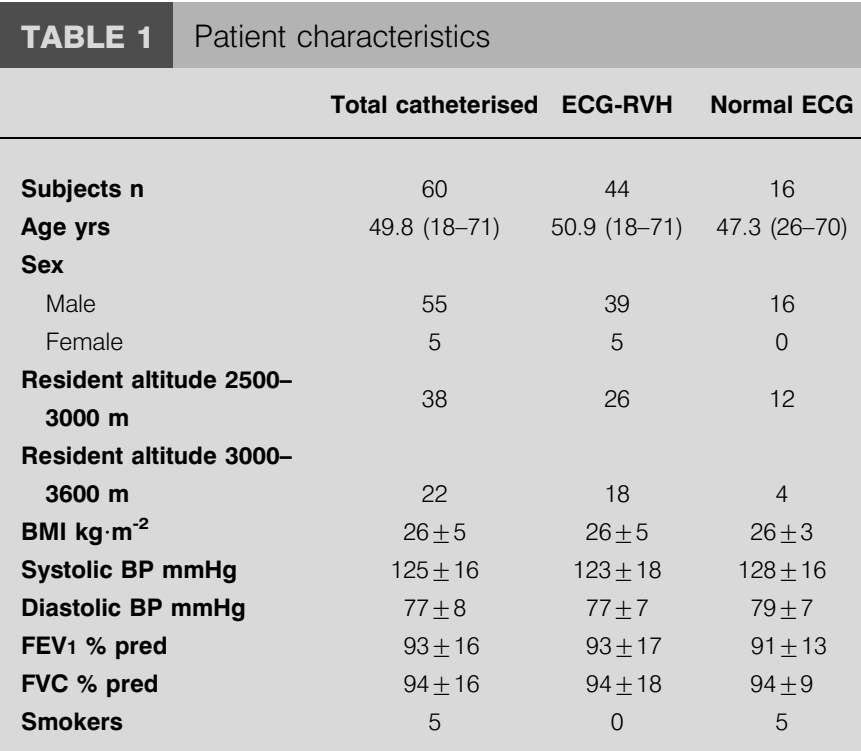

Data are presented as $n$, median (range) or mean \pm SD. ECG-RVH: right ventricular hypertrophy on ECG; BMI: body mass index; BP: blood pressure; FEV1: forced expiratory volume in one second; \% pred: \% predicted; FVC: forced vital capacity. 


\section{Study protocol}

Thus, 60 Kyrgyz highlanders were recruited, 44 with ECGRVH and 16 with a normal ECG; all with no other clinical or spirometric evidence of cardiovascular or respiratory disease. The sensitivity and specificity of the ECG for the detection of RVH was evaluated on the basis of echocardiographic measurement of right ventricular wall thickness in all the subjects. The sensitivity and specificity of Doppler echocardiography for the detection of pulmonary hypertension was evaluated on the basis of invasive measurements of $P$ pa.

\section{Statistical analyses}

Results are expressed as mean \pm SD. $\mathrm{P}<0.05$ was taken as evidence of significance. An unpaired t-test was used for the comparisons between groups. A paired t-test was used for comparisons within groups. Data from catheterisation and echocardiography studies were examined using Spearman and Bland-Altman analyses [9]. The performance of ECG data with respect to the diagnosis of $\mathrm{RVH}$ and $\mathrm{HAPH}$, and echocardiography data in the diagnosis of HAPH was assessed by calculating sensitivity (true positives/(true positives+false negatives)), specificity (true negatives/(true negatives+false positives)), and positive (true positives/(true positives+false positives)) and negative predictive values (true negatives/ (true negatives+false negatives)).

\section{RESULTS}

\section{ECG}

Of the 1,430 high-altitude residents (age $38 \pm 1$ yrs; 660 male) screened, 412 (29\%; 290 male) subjects had one or more ECGRVH pattern. Pattern A was observed in 124 (8.7\%) subjects, pattern B in $11(0.8 \%)$ and pattern $C$ in 167 (11.6\%; fig. 1). Additionally, $86(6 \%)$ subjects had a combination of patterns $\mathrm{A}$ and $\mathrm{C}$, three $(0.2 \%)$ had a combination of patterns $\mathrm{A}$ and $\mathrm{B}$ and $21(1.5 \%)$ had a combination of patterns A, B and C. The prevalence of ECG-RVH was related to the altitude of residency; $23.3 \%$ of subjects living at altitudes $2,500-3,000 \mathrm{~m}$ and $55.3 \%$ of subjects residing higher than $3,000 \mathrm{~m}$ above sea level had ECG-RVH $(\mathrm{p}<0.01)$.

\section{Echocardiography study}

Although tricuspid regurgitation was identified in 42 out of 60 $(69 \%)$ subjects, a signal of sufficient quality for unequivocal reading of a maximum velocity was recovered in only 17 (28 $\%$ ) of them. On the other hand, a satisfactory pulmonary flow velocity signal was recovered in all $60(100 \%)$ subjects. Therefore, only the acceleration time of pulmonary blood flow velocity was used for the estimation of $P$ pa. Calculated mean $P$ pa ranged 14-44 $\mathrm{mmHg}$ (mean $P$ pa $26 \pm 7 \mathrm{mmHg}$ ). In total, 37 subjects were considered to have HAPH based on a mean $P$ pa $\geqslant 25 \mathrm{mmHg}$ and 23 were regarded as normal (table 2). Right ventricular dimensions and right ventricular anterior wall thickness in patients with HAPH were significantly greater than in normal subjects $(2.6 \pm 0.6$ versus $2.1 \pm 0.5 \mathrm{~cm} ; \mathrm{p}<0.001$ and $0.5 \pm 0.1$ versus $0.4 \pm 0.05 \mathrm{~cm} ; \mathrm{p}<0.001)$.

\section{Right heart catheterisation study}

Mean $P$ pa measured at right heart catheterisation ranged 15$54 \mathrm{mmHg}(26 \pm 8 \mathrm{mmHg})$. Mean $P$ pa was $\geqslant 25 \mathrm{mmHg}$ in 29 highlanders and was normal in 31 (table 3). Mean age in the group diagnosed with $\mathrm{HAPH}$ was higher than in the normal

\begin{tabular}{|c|c|c|}
\hline Parameters & HAPH & Normal \\
\hline Subjects $\mathbf{n}$ & 37 & 23 \\
\hline AcT $\mathrm{ms}$ & $91 \pm 3$ & $120 \pm 2^{\#}$ \\
\hline Ppa $\mathrm{mmHg}$ & $30 \pm 6$ & $19 \pm 3^{\#}$ \\
\hline fc beats $\mathrm{min}^{-1}$ & $68 \pm 10$ & $64 \pm 8$ \\
\hline
\end{tabular}

group $(\mathrm{p}<0.001)$. Mean and systolic $P$ pa and pulmonary vascular resistance (PVR) were also significantly higher in $\mathrm{HAPH}$ patients compared with the normal controls $(\mathrm{p}<0.001)$. Cardiac output and frequency did not significantly differ between these two groups.

\section{Sensitivity and specificity of ECG in diagnosis of RVH and HAPH}

In 44 subjects with one or more ECG-RVH patterns, the right ventricular anterior wall thickness was $>0.5 \mathrm{~cm}$ on echocardiographic study in 22 subjects, giving an ECG sensitivity for diagnosing $\mathrm{RVH}$ of $50 \%$. In 16 subjects with a normal ECG, the right ventricular wall thickness was normal $(0.4 \pm 0.05 \mathrm{~cm})$, giving a specificity of $100 \%$. Negative predictive value by ECG was $42 \%$ and positive predictive value was $100 \%$.

In 44 subjects with ECG-defined RVH, pulmonary hypertension was confirmed by direct invasive measurement in 26 subjects. Thus, the sensitivity of ECG for detecting HAPH was $59 \%$. In 16 subjects with a normal ECG, pulmonary hypertension was diagnosed at right heart catheterisation in three subjects, giving a specificity for ECG of $81 \%$. Negative predictive value of ECG was $42 \%$ and positive predictive value was $90 \%$.

TABLE 3 Right heart catheterisation study

\begin{tabular}{|c|c|c|}
\hline Parameters & HAPH & Normal \\
\hline Subjects $\mathrm{n}$ & 29 & 31 \\
\hline Age yrs & $58.3 \pm 9.8$ & $41.7 \pm 15.9^{\#}$ \\
\hline Ppa $\mathrm{mmHg}$ & $33 \pm 7$ & $20 \pm 3^{\#}$ \\
\hline Systolic Ppa mmHg & $49 \pm 11$ & $32 \pm 5^{\#}$ \\
\hline PVR dyne $\cdot \sec ^{-1} \cdot \mathbf{c m}^{-5}$ & $374 \pm 159$ & $194 \pm 56^{\#}$ \\
\hline $\mathrm{CO} L \cdot \min ^{-1}$ & $5.4 \pm 1.2$ & $5.3 \pm 1.2$ \\
\hline fc beats $\mathrm{min}^{-1}$ & $67 \pm 9$ & $66 \pm 11$ \\
\hline
\end{tabular}

Data are presented as $\mathrm{n}$ or mean $\pm \mathrm{SD}$. HAPH: high-altitude pulmonary hypertension; Ppa: pulmonary arterial pressure; PVR: pulmonary vascular resistance; $\mathrm{CO}$ : cardiac output; $f \mathrm{C}$ : cardiac frequency. ${ }^{\#}: \mathrm{p}<0.001$ compared with $\mathrm{HAPH}$ subjects. 

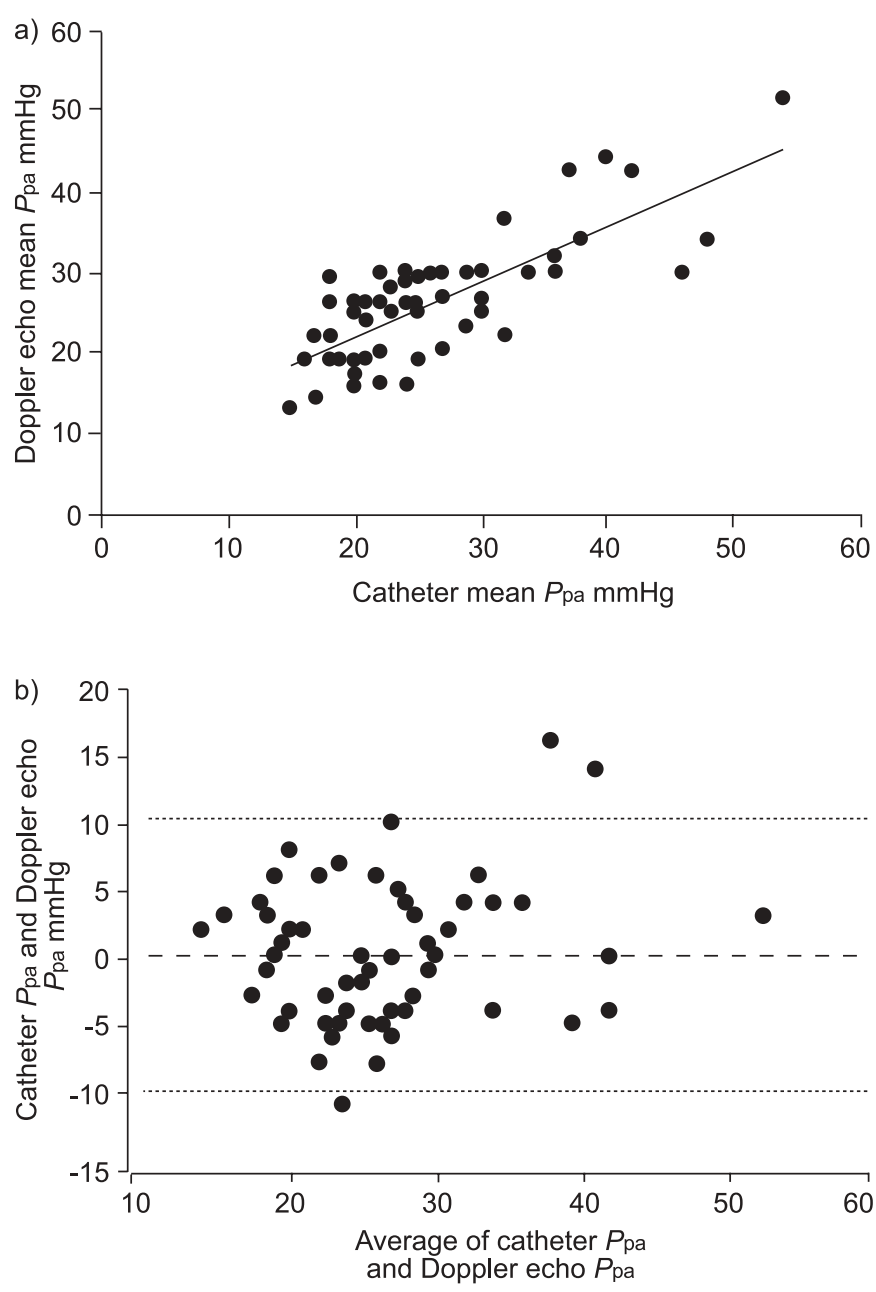

FIGURE 2. a) Correlation between mean pulmonary arterial pressure $P$ pa estimated by Doppler echocardiography (Doppler echo $P$ pa) and mean $P$ pa measured invasively by right heart catheterisation (catheter $P$ pa). The correlation coefficient between echocardiography and catheterisation studies was $r^{2}=0.78$ $(-$. b) Bland-Altman plot of the same data. --- : mean; ……..: $\pm 1.96 \mathrm{sD}$.

\section{Sensitivity and specificity of echocardiography in diagnosis of $\mathrm{HAPH}$}

A total of 12 subjects from the pulmonary hypertension group diagnosed at echocardiography (echocardiography mean $P$ pa $27 \pm 2 \mathrm{mmHg}$ ) had a normal mean $P$ pa on cardiac catheterisation $(22 \pm 2 \mathrm{mmHg} ; \mathrm{p}<0.001)$. Four subjects with normal mean $P$ pa on echocardiography $(21 \pm 2 \mathrm{mmHg})$ had pulmonary hypertension on direct measurement (mean $P$ pa $28 \pm 3 \mathrm{mmHg} ; \mathrm{p}<0.005$ ). Thus, the sensitivity of echocardiography for $\mathrm{HAPH}$ was $70 \%$ and the specificity was $88 \%$. The negative predictive value of echocardiography was $72 \%$ and the positive predictive value was $88 \%$. The correlation coefficient between echocardiography and catheterisation studies was $\mathrm{r}^{2}=0.78$, and the Bland-Altman analysis showed a satisfactory agreement between the two methods of measurement (fig. 2).

\section{DISCUSSION}

The present results show that a combination of clinical examination, ECG and echocardiography can be useful for noninvasive screening for pulmonary hypertension in highaltitude populations.

Ppa can be estimated from Doppler echocardiographic measurements of tricuspid regurgitation or pulmonary flow waves [10]. The most commonly used echocardiographic method relies on the measurement of maximum velocity of tricuspid regurgitation and calculating the trans-tricuspid pressure gradient using the simplified form of the Bernouilli equation:

$\triangle \mathrm{PRV}-\mathrm{RA}=4(V \mathrm{TR})^{2}$

where $\Delta \mathrm{P}$ is pressure difference, $\mathrm{RV}$ the right ventricle, $\mathrm{RA}$ the right atrium and $V$ TR the maximum velocity of tricuspid regurgitation [6]. Systolic $P$ pa is then estimated by adding the clinically determined mean jugular venous pressure to the pressure gradient between the right ventricle and atrium [6]. This method has been widely used in patients with a variety of cardiac diseases, with reported correlation coefficients between Doppler and catheter measurements ranging 0.89-0.97, accurate prediction of $P$ pa over a wide range of pressures and low interobserver variability [10]. A good agreement between catheter and Doppler tricuspid regurgitation measurements of systolic $P$ pa has even been reported in high-altitude environments [11]. However, as illustrated by the present study, the recovery rate of sufficient quality Doppler velocity envelopes for an accurate determination of maximum velocity of regurgitation decreases with the severity of pulmonary hypertension, down to $24-50 \%$ in normal subjects and in patients with mild pulmonary hypertension secondary to chronic lung diseases [10]. This may be the reason for a high proportion of false positives and negatives reported in screening programmes for pulmonary hypertension using Doppler studies of tricuspid regurgitation [12].

The alternative, though less popular, Doppler echocardiographic method for the estimation of $P_{\mathrm{pa}}$ is based on the analysis of the morphology of pulmonary artery flow waves [7]. The acceleration time of pulmonary flow has been repeatedly showed to be inversely correlated to mean $P$ pa, with correlation coefficients ranging $0.65-0.96$ in a variety of cardiac and pulmonary conditions [10]. The intraobserver and interobserver variabilities and errors on the estimate are somewhat higher than on the maximum velocities of tricuspid regurgitation, but the recovery rate of adequate signals is higher, in the range $81-98 \%$, even in patients with chronic obstructive lung disease [10], and may attain 100\% in normal subjects [13]. Acceleration time is affected by heart rate, but this only matters outside the normal range: $<60 \cdot \mathrm{min}^{-1}$ or $>100 \cdot \mathrm{min}^{-1}[14]$, which did not occur in the present study. In the present selected group of altitude inhabitants, the recovery rate of adequate quality Doppler pulmonary artery flow waves was $100 \%$, and there was a correlation of $r^{2}=0.78$, showing a good agreement with invasively measured mean $P$ pa. The sensitivities and specificities of 70 and $88 \%$, respectively, appear to be adequate for a screening programme, although admittedly this should be systematically evaluated on a larger patient population than in the present investigation.

A recognised limitation of the study is that the echocardiography and catheterisations were performed within the first 7 days after the patients were brought down to $760 \mathrm{~m}$. This would certainly reverse hypoxia-induced increase in pulmonary vascular tone, 
and less so pulmonary vascular remodelling, as indicated by persistently increased $P$ pa in the patients with HAPH. The current authors assume that the agreement between echocardiographic and catheter measurements observed at $760 \mathrm{~m}$ would stay the same at higher altitudes.

It is important to emphasise that the present study also relied on ECG as a screening tool for high-altitude pulmonary hypertension. The ECG is affected by right ventricular hypertrophy, itself a consequence of sustained elevation in pulmonary arterial pressure. The ECG is generally believed to be insensitive to the presence of pulmonary hypertension, as reported sensitivities have been as low as $20 \%$ [8]. In the present study, the sensitivity of the ECG for the detection of right ventricular hypertrophy was $50 \%$, but the specificity was $100 \%$. As for the detection of catheter-confirmed pulmonary hypertension, the sensitivity and specificity of the ECG were 59 and $81 \%$, respectively. These numbers compare favourably with general belief that ECG cannot be used for the screening for pulmonary hypertension. Therefore, it may be worth reevaluating the performance of ECG, both alone and in combination with echocardiography, in larger numbers of patients at risk of pulmonary hypertension.

\section{REFERENCES}

1 Mirrakhimov M, Winslow R. The cardiovascular system at high altitude. In: Frigley M, Blatteal C, eds. Environmental Physiology. Oxford, Oxford University Press, 1996; pp. 1241-1258.

2 Penaloza D, Arias-Stella J, Sime F, Reeavarren S, Marticorena E. The heart and pulmonary circulation in children at high altitudes: physiology, anatomical and clinical observations. Pediatrics 1964; 34: 568-582.

3 Aldashev AA. High altitude pulmonary hypertension and signal transduction in the cardiovascular system. J Recept Signal Transduct Res 2000; 20: 255-278.

4 Aldashev AA, Sarybaev ASh, Sydykov AS, et al. Characterization of high-altitude pulmonary hypertension in the
Kyrgyz: association with angiotensin-converting enzyme genotype. Am J Respir Crit Care Med 2002; 166: 1396-1402.

5 Prineas RJ, Crow RS, Blackburn H. The Minnesota Code Manual of Electrocardiographic Findings. Standards and Procedures for Measurement and Classification. Bristol, John Wright, 1982.

6 Yock PG, Popp RL. Noninvasive estimation of right ventricular systolic pressure by Doppler ultrasound in patients with tricuspid regurgitation. Circulation 1984; 70: 657-662.

7 Kitabatake A, Inoue M, Asao M, et al. Noninvasive evaluation of pulmonary hypertension by a pulsed Doppler technique. Circulation 1983; 68: 302-309.

8 Chou T-C, Knilans TK. Electrocardiography in Clinical Practice, 4th Edn. Philadelphia, W.B. Saunders, 1996.

9 Bland JM, Altman DG. Measuring agreement in method comparison studies. Stat Methods Med Res 1999; 8: 135-160.

10 Naeije R, Torbicki A. More on the noninvasive diagnosis of pulmonary hypertension: Doppler echocardiography revisited. Eur Respir J 1995; 8: 1445-1449.

11 Allemann Y, Sartori C, Lepori M, et al. Echocardiographic and invasive measurements of pulmonary artery pressure correlate closely at high altitude. Am J Physiol Heart Circ Physiol 2000; 279: H2013-H2016.

12 Denton CP, Cailes JB, Phillips GD, Wells AU, Black CM, Bois RM. Comparison of Doppler echocardiography and right heart catheterization to assess pulmonary hypertension in systemic sclerosis. Br J Rheumatol 1997; 36: 239-243.

13 Vachiery JL, McDonagh $\mathrm{T}$, Moraine JJ, et al. Doppler assessment of hypoxic pulmonary vasoconstriction and susceptibility to high-altitude pulmonary oedema. Thorax 1995; 50: 22-27.

14 Chan KL, Currie PJ, Seward JB, Hagler DJ, Mair DD, Tajik AJ. Comparision of three Doppler ultrasound methods in the prediction of pulmonary artery pressure. J Am Coll Cardiol 1987; 9: 549-554. 